\title{
Endothelial progenitor cells in debate
}

Cell Research (2006) 16:529. doi:10.1038/sj.cr.7310079; published online 15 June 2006

Adult stem/progenitor cells play important roles in tissue homeostasis and have important implications for regenerative medicine. It was once thought that formation of new blood vessels in adult only occurs through angiogenesis, a process whereby new vessels are formed from existing mature endothelial cells; while vasculogenesis, where new vessels are derived from differentiation of endothelial progenitor cells (EPCs), was thought to occur exclusively in embryos. The discovery of adult EPCs a few years ago has changed this old paradigm; and subsequent studies showed that EPCs may be a promising tool for the treatment of vascular disorders. However, there have been conflicting reports on subtypes, surface markers, and functions of EPCs; and thus the exact origin and identity of EPCs remain to be defined. A common approach to obtain EPCs is to isolate and culture mononuclear cells from peripheral blood and to select adherent cells for further analyses. In the June issue of Cell Research, Zhang et al. attempted to isolate putative "EPCs" using this approach, and examined the phenotypes of these cells in subsequent culture spanning several weeks. Unexpectedly, they found that these putative EPCs exhibited very little proliferative capacity. Marker analyses showed that these cells express some of the endothelial markers (such as KDR and VE-cadherin) as well as the monocyte-specific marker CD14. However, these cells performed poorly in endothelial functional assays in vitro, as they did not give rise to any tube-like structures. Moreover, the authors performed phagocytosis tests and found that these cells maintained their monocyte/macrophagelike function throughout the in vitro culturing process. The authors conclude that although they could obtain putative "EPCs" exhibiting both endothelial and monocyte characteristics from peripheral blood, these cells do not appear to perform EPC functions. Thus, the study by Zhang et al. questions the identity of "EPCs" in peripheral blood, and adds to the ongoing debate concerning the origin of "true" EPCs.

Dangsheng Li

\section{References and links}

\section{Original article}

Shi Ju Zhang, Hao Zhang, Ying Jie Wei, Wen Jun Su, Zhong Kai Liao, Mai Hou, Jian Ye Zhou, Sheng Shou Hu. Adult endothelial progenitor cells from human peripheral blood maintain monocyte/macrophage function throughout in vitro culture. Cell Res 2006; 16:577-584. 